\title{
Spectral and Variational Analysis of Generalized Cylindrical and Elliptical Strip and Microstrip Lines
}

\author{
FRANCISCO MEDINA AND MANUEL HORNO, MEMBER, IEEE
}

\begin{abstract}
In this paper, the variational technique in the spectral domain (VTSD) is shown to be an efficient method for computing the quasi-TEM parameters of arbitrary multiconductor and multidielectric cylindrical or elliptical strip configurations. Simple conformal mappings reduce the cylindrical or elliptical geometries to an equivalent rectangular one with periodic boundary conditions. Minor modifications of previous work on planar structures allow us to analyze any cylindrical or elliptical geometry, computing the capacitance $[C]$ and inductance $[L]$ matrices, from which the effective dielectric constants and mode impedances are obtained.
\end{abstract}

\section{INTRODUCTION}

$I^{N}$ N THE RECENT microwave literature, several papers have been devoted to the analysis of striplike and microstriplike transmission lines printed on flexible dielectrics wrapped around cylindrical or elliptical surfaces. These systems can be used to excite conformal arrays mounted on cylindrical or elliptical objects. Coaxial-toplanar line transitions, slotted lines, and warping due to severe environmental changes can also be conveniently modeled with cylindrical strips.

A number of authors have studied cylindrical and elliptical configurations using the quasi-TEM model. Wang uses a dual series representation to analyze the homogeneous cylindrical stripline and the inhomogeneous cylindrical microstrip [1]. The modified residue calculus technique is used by Joshi et al. to solve cylindrical and elliptical striplines [2]. Conformal mappings have been applied to several simple configurations [3]-[6] and, very recently, the more involved multiconductor striplinelike problem [7]. The Green's function formulation with variational expressions for the capacitance is used to analyze elliptic single [8] and coupled [9] arc strips and a nonsymmetrical pair of coupled cylindrical strips [10]. Single [11]

Manuscript received August 29, 1989; revised April 6, 1990. This work was supported by DGICYT (Project PB87-0798-C03-01, 1988-91) and by the Junta de Andalucía (Project P5355-2, 1988-90).

The authors are with the Departmento de Electrónica y Electromagnetismo, Facultad de Física, Universidad de Sevilla, 41012, Sevilla, Spain.

IEEE Log Number 9036755. and broad-side-coupled [12] cylindrical strips have been analyzed solving the Laplace equation subject to the appropriate boundary conditions in a three-dielectric medium. The spectral-domain technique in cylindrical coordinates has been used to analyze single and symmetrically coupled arc strips [13]. A significant step in the analysis of systems of this kind has been given in [14], where a general class of multiconductor cylindrical lines was studied by taking advantage of the periodicity of these structures and using the FFT algorithm in conjunction with an iterative scheme. In addition, a full-wave analysis of single and coupled cylindrical strips printed on one-layer substrate has been reported in [15] and [16].

The aim of the present paper is to show that generalized cylindrical or confocal elliptical strip geometries (Fig. 1(a)) can be easily studied by using the theory and computer programs previously developed for planar structures with rectangular boundary conditions. Note that most of the transmission lines mentioned in the previous paragraph can be viewed as particular cases of this generic multiconductor system. The method is based on the application of conformal mappings which transform the cylindrical or elliptical geometry into a planar one with rectangular periodic boundary conditions. This equivalent structure is analyzed by using the VTSD approach described in [17], [18] merely introducing slight modifications in the analytical treatment and the computer programs. This analysis allows one to compute the $[L]$ and $[C]$ matrices characterizing the system under quasi-TEM operation and, from these, the modal impedances and effective dielectric constants. The original programs based on [17] and [18] have been improved by using better trial functions and a convenient asymptotic analysis in order to accelerate the convergence of the series appearing in the numerical computation. In this way, we have a very efficient algorithm to characterize, under the quasi-TEM assumption, very general cylindrical or confocal elliptical transmission systems involving an arbitrary number of dielectric layers and conductors distributed between them, even if they are printed in different interfaces. 


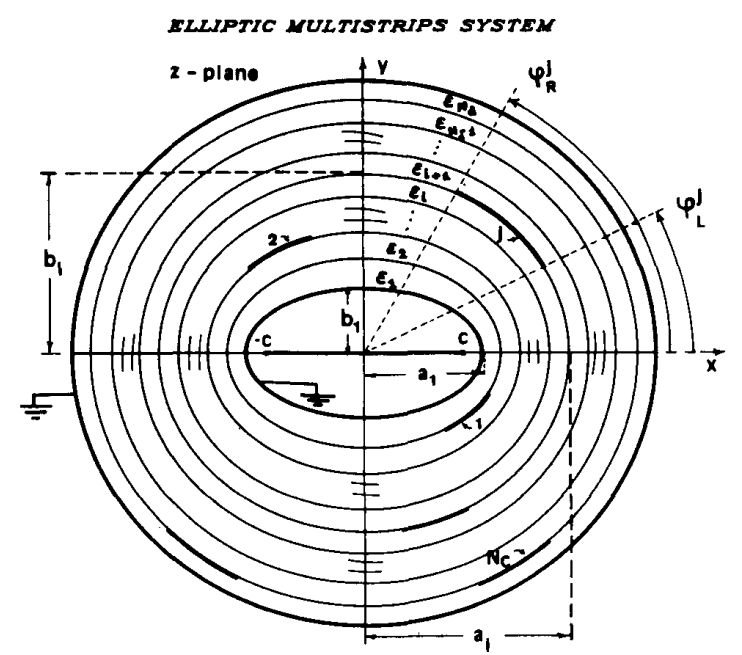

(a)

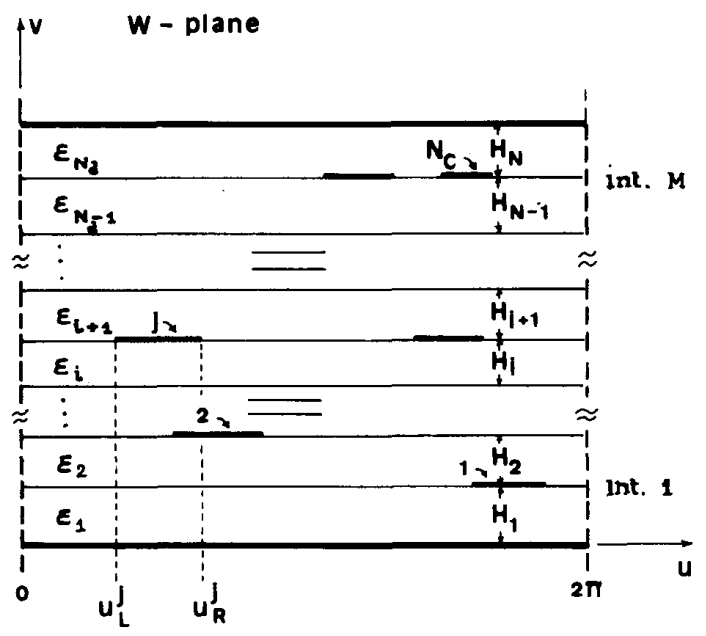

(b)

Fig. 1. (a) Cross section of generalized elliptical multistrip and multidielectric structure. The notation for geometrical parameters defining arc strips and interfaces is included. When $c=0, a_{i}=b_{i}=r_{i}$, we have the cylindrical case. (b) Image structure in $w$ plane of the configuration in (a). This structure is periodic in the $u$ direction with period $2 \pi$.

\section{General Configurations and CONFORMAL MAPPINGS}

The cross section of a generalized confocal elliptical system has been drawn in Fig. 1(a). Our purpose is to compute the $[L]$ and $[C]$ matrices of this multiconductor transmission line. Instead of working in an elliptical frame, we will exploit the theory and the programs developed by the authors for treating generalized planar configurations [17], [18]. In order to do this, we transform the original elliptical (Fig. 1(a)) geometry into an equivalent one with periodic rectangular boundary conditions (Fig. 1(b)) via the following conformal mapping:

$$
w(z)=\cos ^{-1}\left(z^{*} / c\right)-j \tan ^{-1}\left(b_{1} / a_{1}\right)
$$

where the asterisk denotes complex conjugate; $2 c=$ focal distance; $z=x+j y ; w=u+j v$; and $b_{1}$ and $a_{1}$ are the minor and major semiaxes of inner ground ellipse.

The original elliptical configuration in the $z$ plane is mapped into the rectangular one in the $w$ plane. With the mapping given by (1), geometrical parameters (a) and (b) of Fig. 1 are related in the following way:

$$
\begin{aligned}
H_{i} & =\tanh ^{-1}\left(b_{i+1} / a_{i+1}\right)-\tanh ^{-1}\left(b_{i} / a_{i}\right) \\
u_{L}^{j} & =\tan ^{-1}\left[\left(a_{i} / b_{i}\right) \tan \varphi_{L}^{j}\right] \\
u_{R}^{j} & =\tan ^{-1}\left[\left(a_{i} / b_{i}\right) \tan \varphi_{R}^{j}\right]
\end{aligned}
$$

(using the notation in Fig. 1(a) and (b)).

For a cylindrical configuration in the $z$ plane (that is, $c=0, a_{i}=b_{i}=r_{i}$ in Fig. 1(a)) a suitable mapping function is

$$
w(z)=j \ln \left(z^{*} / a\right) .
$$

In this way the cylindrical system is transformed into the rectangular one shown in Fig. 1(b):

$$
H_{i}=\ln \left(r_{i-1} / r_{i}\right) \quad u_{L}^{j}=\varphi_{L}^{j} \quad u_{R}^{j}=\varphi_{R}^{j} .
$$

The equivalent configuration (Fig. 1(b)) consists of an arbitrary number $\left(N_{c}\right)$ of conducting strips lying in a certain number $(M)$ of planar interfaces between $N_{d}$ lossless isotropic dielectric layers, the whole being enclosed by a set of rectangular boundary conditions. The cylindrical or elliptical configuration and their corresponding transformed structures have the same $[C]$ and $[L]$ matrices (nonmagnetic materials are assumed). A generic configuration similar to the one in Fig. 1(b) has been efficiently analyzed by the authors using the variational technique in the spectral domain in [17] and [18]. Since the analysis is parallel to the one reported in [17] and [18], we refer the reader to these papers and to the Appendix herein for details. A brief account will be given in the following section.

\section{Numerical Results}

The $[C]$ matrix is computed from the electrical energy stored per unit length in the structure for an appropriate set of excitations of the conductor strips [18]. The unknown surface charge distribution on the strips for each excitation is expanded into a set of basis functions and the expansion coefficients are computed to minimize the electrical energy (Rayleigh-Ritz method). In order to do this, we work in the Fourier transform domain, since the spectral Green's function matrix (SGFM) needed in the analysis can be easily obtained using the method reported in [17]. As is well known, $[L]$ is obtained from the $[C]$ matrix for the same structure without dielectrics.

The numerical efficiency of the Rayleigh-Ritz algorithm strongly depends on the choice of trial functions for approximating the unknown surface charge density on the strips. In the present work we use a set of basis functions which has proved to be very good for both quasi-TEM 
and full-wave analyses because they incorporate the singular behavior of the surface charge density $(\rho)$ at the strip edges and the properties of Chebyshev polynominals:

$$
\begin{gathered}
\rho^{j}(u)=\rho_{0}^{j}(u)+\sum_{m=1}^{n f} a_{m}^{j} \rho_{m}^{j}(u) \\
\rho_{m}^{j}(u)=\left(2 / \pi W^{j}\right) T_{m}(t) / \sqrt{1-t^{2}} \quad t=\frac{\left[u-S^{j} / 2\right]}{W^{j} / 2} \\
S^{j}=\left(u_{L}^{j}+u_{R}^{j}\right) / 2 \quad W^{j}=u_{R}^{j}-u_{L}^{j}
\end{gathered}
$$

where $T_{m}(t)$ is a Chebyshev polynominal of the first kind of order $m$.

At this point, some attention must be given to the numerical convergence of Fourier series appearing in the analysis (see [18, eqs. (8)-(10)]). The series arising from interactions between noncoplanar strips are quickly convergent, because the off-diagonal elements of the SGFM relating the Fourier transform of the potential and the surface charge in different interfaces decrease exponentially. However, interactions between strips lying at the same interface yield poorly convergent series (if the basis functions in (5) are used, the convergence is as $1 / n^{2}$ ). A significant improvement is achieved if the asymptotic tail extraction technique is applied (see the Appendix). In Fig. 2, we show a typical convergence pattern with and without asymptotic extraction. Clearly, asymptotic extraction will result in an important reduction of CPU time. This fact is even more pronounced for multiple-strip configurations, and it has been verified for a wide variety of simple and coupled structures.

On the basis of this theory we have written a computer program whose reliability and numerical efficiency have been conveniently tested. Table I shows the convergence of the normalized characteristic impedance $\left(\epsilon_{r}^{1 / 2} z_{0}\right)$ with the number of trial functions for a cylindrical homogeneous stripline. We have included some results tabulated in [14, table I, $N=2048$ ] for comparison. The particular case $\alpha=180$, which can be exactly solved by conformal mapping (exact value $=5.361 \Omega$ ), has also been included. The agreement with data reported in [14] is excellent, and the exact value is also obtained. We can see from this table that bigger angles require more basis functions. Nevertheless, CPU time does not increase as quickly with the arc width, since bigger angles require adding fewer Fourier terms. Typically, four significant digits are obtained in $50-200 \mathrm{~ms}$ on a VAX-11/785 computer. Note that our method does not impose restrictions on the strip width, in contrast to the method in [14], based in the application of the FFT.

The multiconductor case is also checked by comparisons with data reported in [14, table III]. In Table II we present results for the mode effective dielectric constants of six coupled strips. Slight differences could be due to the relatively small number of samples used in [14] to compute these data. In general, very good agreement with data published in [14] has been found. Discrepancies with

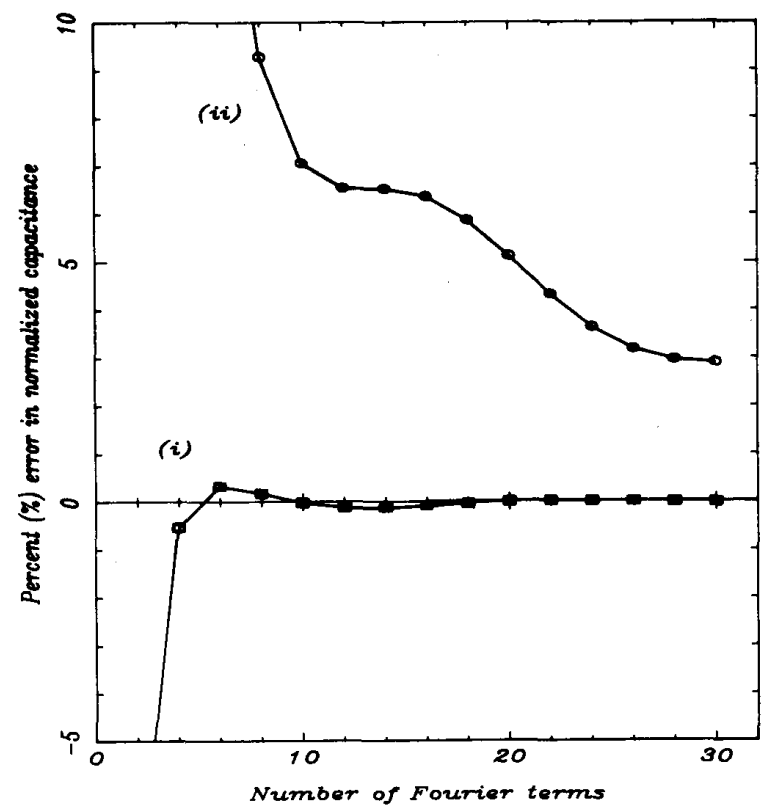

Fig. 2. Percent deviation from the exact value of the normalized capacitance of a cylindrical strip as a function of the number of retained Fourier terms with (i) and without (ii) asymptotic extraction.

graphic data published in [1] and [11] pointed out in [14] have also been detected. Since this disagreement has been found using two completely different methods, we can also conclude that the accuracy of the results in [1] and [11] is questionable.

Edge-coupled symmetrical arc strips have been considered to compare with the results in [5] and [13]. The method in [5] is valid when the geometrical parameters are within a certain range of values. For these, very good agreement is found. However, the authors in [13] use as trial function for the charge density just the zeroth-order term in (5). This choice results in serious numerical errors when strips are strongly coupled. This is because the charge distribution is not symmetrical around the center of the strip, as is assumed in [13]. When wide arc strips are involved, the charge distribution tends to be almost uniform. Thus, significant discrepancies with results in [13] also arise in this case. Table III illustrates these facts. Results in the first column $(n f=0)$ correspond to the method in [13]. In order to obtain accurate results, a few basis functions must be retained. Note that odd trial functions are closely related to the coupling effect, since these take into account the asymmetry of the charge distribution on coupled strips. Even functions must be added when wide strips are involved. Similar accuracy problems are also expected to occur in [8]-[10], where poor trial functions are used.

Noncoplanar strips can be also treated with our program. We have also tested this case. For instance, we have considered the symmetrical broadside-coupled strips analyzed in [6] and [12]. In these papers, the ratio between radii is chosen in such a way that even and odd 
TABLE I

Characteristic Impedance $(\Omega)$ Versus Number of Trial Functions FOR CYLINDRICAL STRIPLine

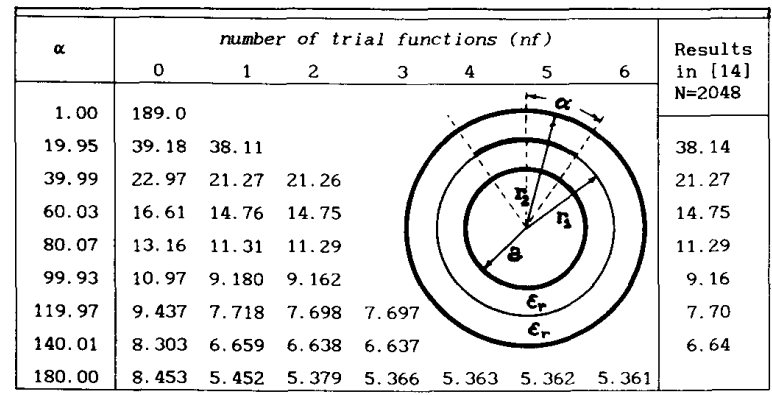

$\epsilon_{r}=1.0 ; r_{1} / a=1.8 ; r_{2} / a=2.0$

TABLE II

Effective Dielectric Constants of Six Coupled Striplines as a Function of Separation Angle

\begin{tabular}{|cc|c|c|c|c|c|c|}
\hline \multirow{2}{*}{$\begin{array}{c}\text { separation } \\
\text { angle } \\
\text { (degrees) }\end{array}$} & \multicolumn{6}{|c|}{ effect ive dielectric constant } \\
\cline { 4 - 8 } & $\begin{array}{c}1 \text { st } \\
\text { mode }\end{array}$ & $\begin{array}{c}2 \text { nd } \\
\text { mode }\end{array}$ & $\begin{array}{c}3 \text { rd } \\
\text { mode }\end{array}$ & $\begin{array}{c}4 \text { th } \\
\text { mode }\end{array}$ & $\begin{array}{c}5 \text { th } \\
\text { mode }\end{array}$ & $\begin{array}{l}6 \text { th } \\
\text { mode }\end{array}$ \\
\hline 0.00 & (a) & 3.0277 & 3.0759 & 3.1405 & 5.0790 & 5.1479 & 5.2594 \\
& (b) & 3.0281 & 3.0765 & 3.1408 & 5.0789 & 5.1487 & 5.2610 \\
\hline 6.33 & (a) & 3.0294 & 3.0777 & 3.1413 & 5.0761 & 5.1457 & 5.2582 \\
& (b) & 3.0299 & 3.0782 & 3.1421 & 5.0755 & 5.1456 & 5.2592 \\
\hline 18.98 & (a) & 3.0362 & 3.0858 & 3.1489 & 5.0619 & 5.1305 & 5.2508 \\
& (b) & 3.0365 & 3.0861 & 3.1496 & 5.0617 & 5.1305 & 5.2507 \\
\hline 35.86 & (a) & 3.0305 & 3.0873 & 3.1622 & 5.0663 & 5.1240 & 5.2470 \\
& (b) & 3.0304 & 3.0868 & 3.1628 & 5.0667 & 5.1252 & 5.2476 \\
\hline 63.98 & (a) & 3.0370 & 3.0885 & 3.1873 & 5.0576 & 5.1133 & 5.2221 \\
& (b) & 3.0379 & 3.0879 & 3.1885 & 5.0585 & 5.1134 & 5.2234 \\
\hline 106.17 & (a) & 3.0372 & 3.1004 & 3.2147 & 5.0562 & 5.1103 & 5.1854 \\
(b) & 3.0375 & 3.1014 & 3.2153 & 5.0569 & 5.1101 & 5.1854 \\
\hline \multirow{2}{*}{6}
\end{tabular}

(a) Results reported in [14, table III]

(b) Our results.

modes can be defined, and the analysis is applied to the single conductor geometry corresponding to each mode. We have analyzed these structures both by considering them as particular cases of the more general asymmetric configuration and by using the theory of odd and even modes, introducing the appropriate electric or magnetic wall [12]. The numerical results obtained with both procedures are indistinguishable. Very good agreement has been found with the conformal mapping results in [6] for a wide range of values of $\alpha$. However, slight discrepancies with the variational results in [12] were found for high values of $\alpha$. This is due again to a lack of precision in the estimation of charge distribution, as is evident from Fig. 3. In this figure, we show the ratio of phase velocities for broadside-coupled microstrip lines using $n f=0$ and $n f=6$ in (5). The computed results coincide with graphic data reported in [12] when $n f=0$, but significant deviation is found ( $10 \%$ for wide strips) when more terms are used to approximate the charge distribution. In the limiting case $\alpha=180$, which can be exactly solved, we get five significant digits using four basis functions in (5).

In order to check the validity of the program for elliptic arc strips, we have compared our data with the very accurate conformal mapping results reported in [3]. These
TABLE III

Even- AND Odd-Mode Characteristic IMPEdance of Edge-Coupled Cylindrical Strips

\begin{tabular}{|c|c|ccccccc|}
\hline \multicolumn{2}{|c|}{ (degrees) } & \multicolumn{6}{|c|}{ number of trial functions } & (nf) \\
\hline$\alpha$ & $\theta$ & 0 & 1 & 2 & 3 & 4 & 5 & 6 \\
\hline \multirow{2}{*}{20} & 2 & 50.92 & 49.89 & 48.80 & 48.77 & 48.77 & 48.77 & \\
& & 37.07 & 35.59 & 35.55 & 35.47 & 35.45 & 35.45 & \\
80 & 2 & 16.79 & 16.63 & 14.29 & 14.27 & 14.26 & 14.25 & 14.25 \\
& & 13.67 & 13.45 & 12.87 & 12.82 & 12.81 & 12.79 & 12.79 \\
& & 45.52 & 45.47 & 45.02 & 45.02 & & & \\
& 10 & 42.47 & 42.41 & 42.23 & 42.23 & & & \\
& & 15.88 & 15.88 & 14.02 & 14.02 & 14.01 & 14.01 & \\
& 10 & 14.58 & 14.58 & 13.46 & 13.46 & 13.46 & 13.46 & \\
& & & & & & & & \\
\end{tabular}

$\mathrm{b} / \mathrm{a}=1.4 ; \mathrm{c} / \mathrm{a}=1.8 ; \varepsilon_{\mathrm{r}}=2.55$

$\mathrm{nf}=0$ corresponds to the technique in [13]
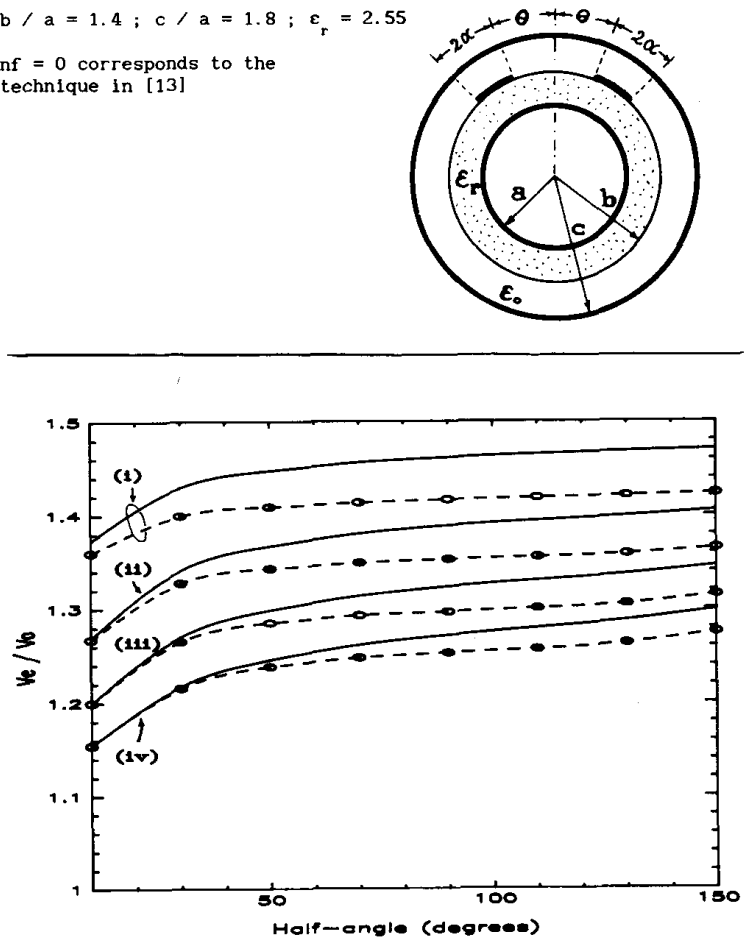

Fig. 3. Variation of mode velocity ratios of broadside-coupled microstrip lines as a function of half-angle strip arc. Notation is the same as that in $[12$, fig. 6]: ----- results in [12]; $\circ$ our results with $n f=0$ in (5); $\longrightarrow$ our results with $n f=6$. (i) $p / a=0.1$, (ii) $p / a=0.3$, (iii) $p / a=0.6$, (iv) $p / a=1.0 . b / a=1.4, \epsilon_{r}=2.32$.

results are limited to single elliptical stripline in homogeneous medium, but they are good enough for our comparison purpose. We have made comparisons with all graphic data reported in [3], and the agreement is excellent in all cases. In Fig. 4, for example, we show the characteristic impedance of an elliptical stripline as a function of the arc width for different locations with respect to the major axis $\left(\theta_{0}\right)$ and two different distances to the inner elliptical ground $(h / a)$. As expected, the characteristic impedance depends on $\theta_{0}$, which enters as a new variable not existing in the cylindrical case.

In conclusion, the computer programs based on the theory in this paper can be used with confidence to compute the characteristic parameters of a very wide variety of cylindrical or elliptical transmission systems. 


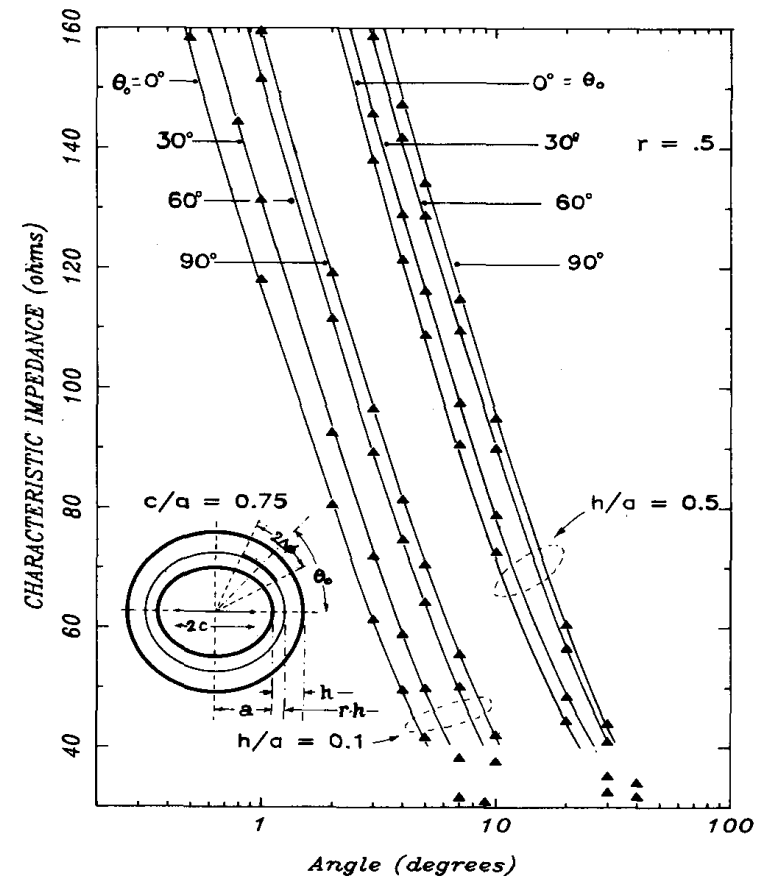

Fig. 4. Characteristic impedances of elliptic stripline in homogeneous medium (air) versus strip angle $(\Delta \phi): \stackrel{-}{\longrightarrow}$ results reported in [3]; $\wedge$ our results.

With the aim of including an example not reported in previous literature, we show in Fig. 5 the effective dielectric constants and mode impedances of a pair of asymmetrical coupled cylindrical strips printed on a two-layer substrate. In Fig. 5(a) we can see that the c- and $\pi$-mode effective dielectric constants can be made almost equal by a proper choice of the thickness of the inner dielectric (which has the lower dielectric constant). The range of values of $(b-a) / a$ where the effective dielectric constants are very close is around a critical point defined by the equalization of the inductive and capacitive couplings. In this zone, the mode line impedances exhibit the singular behavior shown in Fig. 5(b). The $\mathrm{c}$ or $\pi$ nature of each mode changes to each side of the critical point; that is, the corresponding mode number changes the sign. This behavior is similar to that reported in [19] for coupled asymmetric microstrips with overlay. A detailed analysis of these phenomena and an analysis of other particular structures are out of the scope of this paper, which focuses more on the computational method to solve this kind of structure than on the analysis of the behavior of particular configurations.

\section{Conclusions}

In this paper we have stated that very general singleconductor or multiconductor cylindrical/elliptical strip configurations embedded in a layered dielectric medium can be reduced, via conformal mapping, to a rectangular geometry with the same $[L]$ and $[C]$ matrices. The analysis of this equivalent structure is achieved by taking ad-

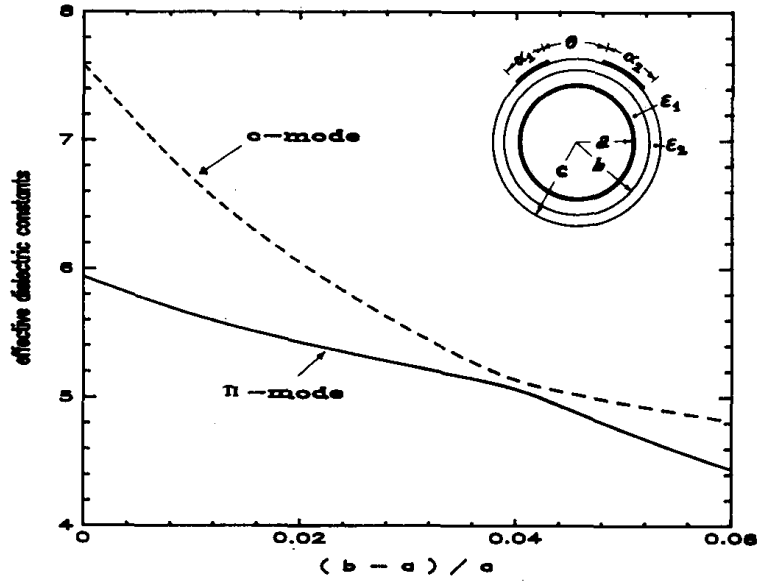

(a)

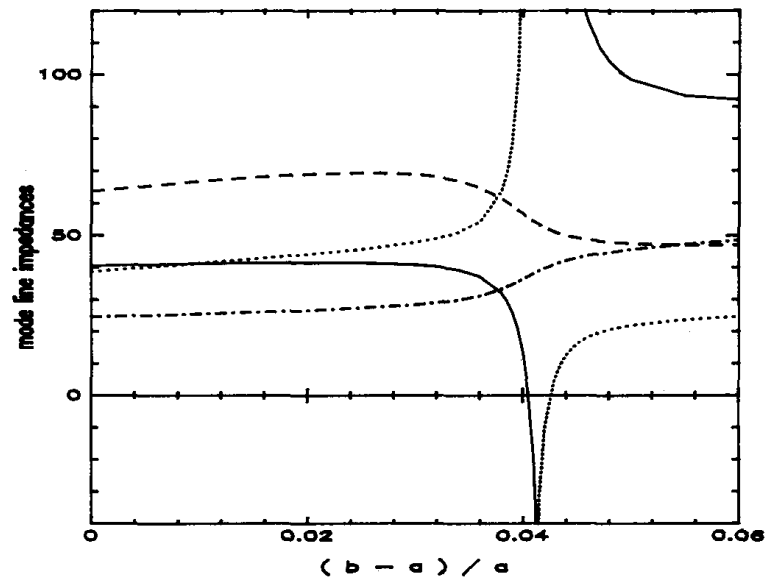

(b)

Fig. 5. (a) Effective dielectric constants of $\mathrm{c}$ and $\pi$ modes of a pair of asymmetric cylindrical strips on two-layer substrate. (b) Modal line impedances of the same structure. Note the singular behaviour around the critical point. $---Z_{1 c} ; \cdots \cdots Z_{1 \pi} ;-Z_{2 c} ; \cdot \cdots \cdot-\cdot$ $Z_{2 \pi} \cdot \alpha_{1}=10^{\circ}, \alpha_{2}=20^{\circ}, \theta=5^{\circ}, c / a=1.2, \epsilon_{1}=2.32, \epsilon_{2}=10$.

vantage of previous work on boxed planar structures. The numerical convergence of the programs is greatly accelerated incorporating the asymptotic behavior of the series appearing in the analysis, in such a way that efficient programs have been written. It has also been pointed out that excessively simple approximations to the surface charge distribution yield meaningful numerical errors, mainly when strong coupling or wide strips are involved. As an application example, the singular behavior of the characteristic parameters of asymmetric coupled structures on multilayer substrates has been shown.

\section{APPENDIX}

Here we include certain remarks on the computation of the spectral Green's function matrix, $\left[\tilde{G}_{i j}(n)\right]$, with $i, j=$ $1, \cdots, M$, used in the VTSD [17], [18] and on numerical aspects concerning the addition of Fourier series involved in the method. 
The structure analyzed in this paper (Fig. 1(b)) differs from the configurations studied in [17] and [18] in the nature of the lateral boundary conditions. The structure in this work is periodic with period $2 \pi$ in the $u$ direction instead of having electric or magnetic sidewalls. Then, all the quantities are Fourier transformed using the definition:

$$
\tilde{F}(n)=\int_{0}^{2 \pi} f(u) e^{j n u} d u .
$$

As a consequence, $\left[\tilde{G}_{i j}(n)\right]^{-1}=\left[\tilde{L}_{i j}(n)\right]$ can be computed from [17, eqs. (2)-(17)] making the following substitutions:

$$
k_{n} \rightarrow n \quad \epsilon_{x x}^{* i}=\epsilon_{y y}^{* i} \longrightarrow \epsilon_{i}
$$

where the subscript $i$ refers to the $i$ th layer in Fig. 1(b), and $\left[\tilde{L}_{i j}(n)\right]$ is a tridiagonal symmetrical matrix with elements defined by

$$
\tilde{L}_{k, k}=\left.\frac{\tilde{\rho}_{k}}{\tilde{V}_{k}}\right|_{\tilde{V}_{k-1}=\bar{V}_{k+1}=0} \quad \tilde{L}_{k, k-1}=\left.\frac{\tilde{\rho}_{k}}{\tilde{V}_{k-1}}\right|_{\tilde{V}_{k}=0}
$$

where $\tilde{\rho}_{k}$ and $\tilde{V}_{k}$ are the Fourier transform of free surface charge and the potential distributions on the $k$ th interface (see Fig. 1(b)). That is, the original boundary condition problem is separated into simpler problems defined by the boundary conditions in (A3). The solutions to these problems are given in [17, eqs. (12)-(17)] in terms of certain $\tilde{g}_{i j}(n)$ functions defined in [17, eq. (6)]. The physical meaning of these functions is evident from [17, eq. (5)]. They are the factors relating the Fourier transform of the free charge density and the potential distributions in the configurations shown in Fig. 6.

On the other hand, all the Fourier series appearing in [17] and [18] must be added from $n=-\infty$ to $n=+\infty$ instead of from $n=1$ to $n=\infty$. In Section III we referred to the need for taking into account the asymptotic behavior of these series in order to achieve good convergence. From [17, eqs. (6) to (17)] we can see that $\left[L_{i j}(n)\right]$ exponentially converges to a diagonal matrix whose elements are

$$
\begin{array}{r}
\tilde{L}_{k, k}(n \rightarrow \infty)=\epsilon_{0}\left(\epsilon_{1}+\epsilon_{i+1}\right)|n|=\tilde{G}_{k, k}^{-1}(n \rightarrow \infty), \\
k=1, \cdots, M
\end{array}
$$

where $i$ and $i+1$ refer to the dielectric layers adjacent to the $k$ th interface

For the basis functions in (5), the Fourier series appearing when the method in [18] is applied has the following form:

$S=\sum_{n=-\infty}^{\infty} A(n)=\sum_{n=-\infty}^{\infty} J_{p}(n a) J_{q}(n b) \tilde{G}_{k, k}(n)\left(\begin{array}{c}\sin (n c) \\ \cos (n c)\end{array}\right)$

where $J_{p, q}$, Bessel functions of the first kind of order $p, q$, are the Fourier transforms of the basis functions in (5).

The convergence of $S$ is not satisfactory. However, if we consider the asymptotic behavior of $A(n)$ when $n \rightarrow \infty$, namely $A_{\infty}(n)$, we can rewrite (A5) as

$$
S=\sum_{n=-\infty}^{\infty}\left[A(n)-A_{\infty}(n)\right]+\sum_{n=-\infty}^{\infty} A_{\infty}(n)
$$

where

$$
A_{\infty}(n)=C \frac{\left[\cos (n a-p \pi / 2-\pi / 4) \cos (n b-q \pi / 2-\pi / 4)\left(\begin{array}{c}
\sin (n c) \\
\cos (n c)
\end{array}\right)\right]}{n^{2}}
$$

with

$$
C=2 /\left[\pi(a b)^{1 / 2} \epsilon_{0}\left(\epsilon_{r}^{i}+\epsilon_{r}^{i+1}\right)\right] .
$$

Now, after some manipulations, the second term in (A6) is expressed as a combination of trigonometric series. One of these series can be expressed in closed form; the other is reduced to an equivalent one converging in a few terms by means of the residue technique. In our programs we have used

$$
\begin{aligned}
& \sum_{n=1}^{\infty} \frac{\cos (n x)}{n^{2}}=\frac{\pi^{2}}{6}-\frac{x(2 \pi-x)}{4}, \quad 0<x<2 \pi \\
& \sum_{n=1}^{\infty} \frac{\sin (n x)}{n^{2}} \simeq \begin{cases}x\left[1-\log (x)+x^{2}\left(\frac{1}{72}+\frac{x^{2}}{14400}\right)\right], & x<0.5 \\
-(2 \pi-x)\left[1-\log (2 \pi-x)+(2 \pi-x)^{2}\left(\frac{1}{72}+\frac{(2 \pi-x)^{2}}{14400}\right)\right], & (2 \pi-x)<0.5 \\
\sum_{n=1}^{7} \frac{\sin (n x)}{n^{2}}[1-\tanh (n)]-\frac{4}{\pi} \sum_{m, \text { odd }}^{\frac{9}{\sinh [m \pi(\pi-x) / 2]}} \frac{m^{2} \sinh \left(m \pi^{2} / 2\right)}{}, & 0.5<x<(2 \pi-0.5)\end{cases}
\end{aligned}
$$

(eight digits being correct in the worst case using these expressions). 


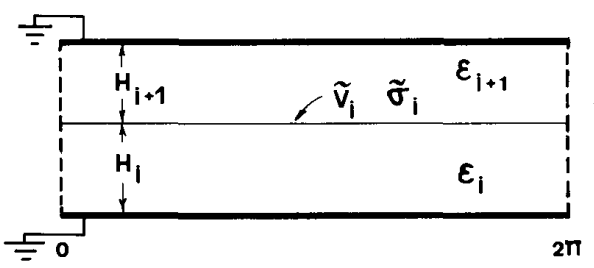

(a)

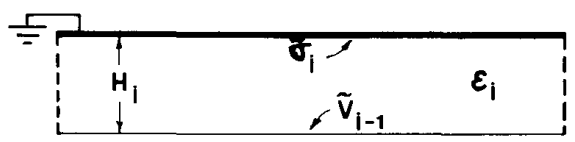

(b)

Fig. 6. Structures defining the elementary spectral functions needed to compute the global matrix Green's function: (a) definition of $\tilde{g}_{i, i}(n)=$ $\tilde{\sigma}_{i} / \tilde{V}_{i} ;$ (b) definition of $\tilde{g}_{i, i-1}(n)=\tilde{\sigma}_{i} / \tilde{V}_{i-1}$. The quantities $\tilde{\sigma}_{i}$ and $\tilde{V}_{i}$ are the Fourier transforms of the free surface charge density and the potential distribution.

\section{REFERENCES}

[1] Y.-C. Wang, "Cylindrical and cylindrically warped strip and microstriplines," IEEE Trans. Microwave Theory Tech., vol. MTT-26, pp. 20-23, Jan. 1978.

[2] K. K. Joshi and B. N. Das, "Analysis of elliptic and cylindrical striplines using Laplace's equation," IEEE Trans. Microwave Theory Tech., vol. MTT-28, pp. 381-386, Apr. 1980.

[3] K. K. Joshi, J. S. Rao, and B. N. Das, "Characteristic impedance of nonplanar striplines," Proc. Inst. Elec. Eng., pt. H, vol. 127, pp. 287-291, Oct. 1980.

[4] L.-R. Zeng and Y. Wang, "Accurate solutions of elliptical and cylindrical striplines and microstriplines," IEEE Trans. Microwave Theory Tech., vol. MTT-34, pp. 259-265, Feb. 1986.

[5] C. J. Reddy and M. D. Deshpande. "Characteristics of inhomogeneous coupled cylindrical striplines," Electron. Lett., vol. 23, no. 16, pp. 821-822, July 1987.

[6] C. J. Reddy and M. D. Deshpande, "Characteristics of broadside coupled cylindrical striplines," Microwave and Opt. Tech. Lett., vol. 1, no. 4, pp. 133-136, June 1988.

[7] D. Homentcovschi, "A cylindrical multiconductor stripline-like microstrip transmission line," IEEE Trans. Microwave Theor Tech., vol. 37, pp. 497-503, Mar. 1989.

[8] B. N. Das, A. Chakraborty, and K. K. Joshi, "Characteristic impedance of elliptic cylindrical strip and microstriplines filled with layered substrate," Proc. Inst. Elec. Eng., pt. H, vol. 130, pp. 245-250, June 1983.

[9] B. N. Das and K. V. S. V. R. Prasad, "Even- and odd-mode impedances of coupled elliptic arc strips," IEEE Trans. Microwave Theory Tech., vol. MTT-32, pp. 1475-1479, Nov. 1984.

[10] C. J. Reddy and M. D. Deshpande, "Analysis of coupled cylindrical striplines filled with multilayered dielectrics," IEEE Trans. Microwave Theory Tech., vol. 36, pp. 1301-1310, Sept. 1988.

[11] C. J. Reddy and M. D. Deshpande, "Analysis of cylindrical stripline with multilayer dielectrics," IEEE Trans. Microwave Theory Tech., vol. MTT-34, pp. 701-706, June 1986.

[12] C. J. Reddy and M. D. Deshpande, "Analysis of broad side coupled inhomogeneous cylindrical striplines," Proc. Inst. Elec. Eng., pt. H, vol. 134, pp. 545-549, Dec. 1987.

[13] M. D. Deshpande and C. J. Reddy, "Spectral domain analysis of single and coupled cylindrical striplines," IEEE Trans. Microwave Theory Tech., vol. MTT-35, pp. 672-675, July 1987.
[14] C. H. Chan and R. Mittra "Analysis of a class of cylindrical multiconductor transmission lines using an iterative approach," IEEE Trans. Microwave Theory Tech., vol. MTT-35, pp. 415-424, Apr. 1987.

[15] N. G. Alexopoulos and A. Nakatani, "Cylindrical substrate microstrip line characterization," IEEE Trans. Microwave Theory Tech., vol. MTT-35, pp. 843-849, Sept. 1988.

[16] A. Nakatani and N. G. Alexopoulos, "Coupled microstrip lines on a cylindrical substrate," IEEE Trans. Microwave Theory Tech., vol. MTT-35, pp. 1392-1398, Dec. 1987.

[17] F. Medina and M. Horno, "Determination of Green's function matrix for multiconductor and anisotropic multidielectric planar transmission lines: A variational approach," IEEE Trans. Microwave Theory Tech., vol. MTT-33, pp. 933-940, Oct. 1985.

[18] F. Medina and M. Horno, "Capitance and inductance matrices for multistrip structures in multilayered anisotropic dielectrics," IEEE Trans. Microwave Theory Tech., vol. MTT-35, pp. 1002-1008, Nov. 1987.

[19] K. Sachse, "Analysis of asymmetrical inhomogeneous coupled-line directional coupler," in Proc. 18th European Microwave Conf., Stockholm (Sweden), Sept. 1988, pp. 985-990.

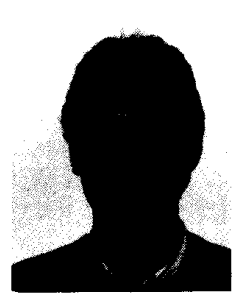

Francisco Medina was born in Puerto Real, Cádiz, Spain, on November 9, 1960. He received the Licenciado degree in September 1983 and the Doctor degree in September 1987, both in physics, from the University of Sevilla, Spain.

$\mathrm{He}$ is currently Assistant Professor of Electricity and Magnetism in the Department of Electronics and Electromagnetism, University of Sevilla. His research focuses mainly on numerical methods for planar structures and multiconductor lines.

斗

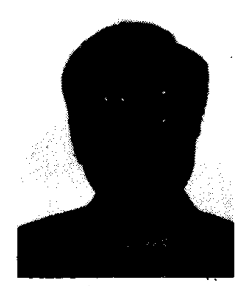

Manuel Horno (M'75) was born in Torre del Campo, Jaén, Spain. He received the degree of Licenciado in physics in June 1969 and the degree of Doctor en Ciencias in physics in January 1972, both from the University of Sevilla, Spain.

Since October 1969 he has been with the Department of Electricity and Electronics at the University of Sevilla, where he became an Assistant Professor in 1970, an Associate Professor in 1975 , and Professor in 1986. His main fields of interest include boundary value problems in electromagnetic theory, wave propagation through anisotropic media, and microwave integrated circuits. He is presently engaged in the analysis of planar transmission lines embedded in anisotropic materials, multiconductor transmission lines, and planar slow-wave structures. 